Ergebnisse einer Metaanalyse

\title{
Trockener Mund durch Medikamente: Harninkontinenzmittel auf Platz 1
}

\section{Xerostomie ist bei älteren Patienten eine häufige Nebenwirkung von Pharma- kotherapien. Bei bestimmten Indikationen ist das Risiko besonders hoch.}

W egen des nachlassenden Speichelflusses nimmt mit dem Alter die Häufigkeit von therapieinduzierter Xerostomie zu. Folgen der Mundtrockenheit sind mehr orale Komplikationen wie Karies und Soor, aber auch häufigere Therapieabbrüche. Ein internationales Ärzteteam um Edwin Tan vom Stockholmer Karolinska-Institut hat daher versucht, das Risiko besonders nebenwirkungsträchtiger Medikamente genauer zu bestimmen.

Eine Literaturrecherche förderte 52 relevante Untersuchungen zutage, darunter 25 placebokontrollierte Studien. Insgesamt korrelierte die Zahl der verordneten Medikamente und Medika- mentenklassen mit dem Risiko für Xerostomie und Hyposalivation.

Medikamente aus drei Indikationen und 22 randomisierten kontrollierten Studien konnten in einer Metaanalyse ausgewertet werden. Dabei erwiesen sich Mittel gegen Harninkontinenz als am stärksten nebenwirkungsbehaftet: Das Risiko für einen trockenen Mund war darunter sechsmal so hoch wie unter Placebo. Innerhalb der Harninkontinenzmedikamente war orales, schnell freisetzendes Oxybutynin mit dem höchsten Risiko verknüpft.

Der Gebrauch von Antidepressiva die meisten Studien wurden mit dem Serotonin-Noradrenalin-Wiederaufname-
Hemmer (SNRI) Duloxetin gemacht ging etwa mit einer Verfünffachung des Xerostomierisikos einher. Tan und Kollgen weisen aber darauf hin, dass anticholinerge Nebenwirkungen unter SNRI und SSRI im Allgemeinen weniger wahrscheinlich sind als unter Trizyklika.

Neuroleptika - untersucht wurden Quetiapin und Eszopiclon - hatten mit plus $60 \%$ den geringsten relativen Risikozuwachs. Absolut war das Risiko für einen trockenen Mund unter Antidepressiva und Neuroleptika um 9\% erhöht.

\section{Medikation regelmäßig prüfen}

Vor allem bei Patienten mit Polypharmazie müsse gut überlegt werden, ob Medikamente, die häufig Mundtrockenheit verursachten, wirklich indiziert seien, so Tan und Kollegen. Wichtig sei, die Medikation regelmäßig auf diese Nebenwirkung hin zu prüfen und ggf. die Dosis zu reduzieren oder die Therapie zu wechseln.

Tan ECK et al. J Am Geriatr Soc 2017, online 26 Oktober 2017; https://dx.doi.org/10.1111/jgs.15151

Prävention

\section{Trainingsprogramm für junge Alte - bald als App}

Eine Smartphone-App soll Menschen ab 60 Jahren helfen, mehr Bewegung in ihren Alltag zu integrieren. Das Konzept wird derzeit in einer Studie geprüft.

$V_{\text {i }}$ or einigen Jahren haben Forscher ein Programm für ältere Menschen entwickelt, das sie zu mehr körperlicher Aktivität bewegen soll. Mit dem „Lifestyle Integrated Functional Exercise Program“, kurz LiFE, ließ sich das Sturzrisiko um etwa ein Drittel senken.

\section{Früher Trainingsbeginn ist besser}

Die Übungen wurden für Menschen über 70 Jahre konzipiert. Am besten wäre es, ältere Menschen würden schon mit dem Training beginnen, bevor sie hinfallen oder ihren Alltag nicht mehr gut bewältigen können. Forscher der Universität Heidelberg versuchen das Programm nun an jüngere Ältere ab 60 Jahre anzupassen. Sie haben eine Version für diese Altersgruppe entwickelt, die sie auch als
App verfügbar machen wollen (eLiFE). Ältere Menschen üben damit u.a., auf Zehenspitzen zu gehen, beim Treppensteigen zwei Stufen auf einmal zu nehmen, auf Spaziergängen Objekte zu überspringen oder auf einem Bein stehend den Oberkörper und das andere Bein waagerecht zu halten. Diese Übungen gibt es in unterschiedlichen Schwierig

Die App-Nutzer können sich mit der Zeit steigern. keitsstufen - die Teilnehmer können sich mit der Zeit also steigern, erläuterte Dr. Michael Schwenk vom Netzwerk Altersforschung in Heidelberg. Die Teilnehmer können ihre Fortschritte mit den Geräten dokumentieren, die App erinnert an das Training und erfasst über Bewegungssensoren die körperliche Aktivität. Derzeit wird das eLiFE-Konzept in einer kontrollierten Studie geprüft.

mut

29. Jahreskongress der Deutschen Gesellschaft für Geriatrie (DGG), 28-30. September, Frankfurt am Main. 